\title{
Manufacturing Technology of Aluminium Wire from Alloy 01417 with Adjusted Level of Mechanical Properties
}

\author{
Yuriy V. Gorokhov, Viktor N. Timofeev, Mikhail V. Pervukhin, \\ Vasiliy I. Belokopytov, Mikhail M. Motkov*, Naydan B. Erdineev, \\ Ivan S. Kosyachenko, Oksana Ya. Yakunina and Alexey S. Strigin \\ Siberian Federal University \\ 79 Svobodny, Krasnoyarsk, 660041, Russia
}

Received 25.09.2019, received in revised form 11.10.2019, accepted 01.11.2019

\begin{abstract}
In the 70s of the last century, Soviet scientists developed an aluminum alloy with $7 \%$ rare earth elements (REE), which at melt cooling speeds of up to $10^{4} \mathrm{deg} / \mathrm{s}$ are dispersed into intermetallic phases, which significantly increase the heat resistance, corrosion resistance, and weldability of finished products for conductive material. To ensure melt cooling rates of up to $10^{4} \mathrm{deg} / \mathrm{s}$ in those years, centrifugation of granules in water was used. To increase the efficiency of this redistribution, a pilot industrial line was used for continuous pellet pressing by the Conform method. A method has been developed for producing small-section billets (A 8-12 $\mathrm{mm}$ ) with a crushed structure from Al-REM system alloys by continuous casting in electromagnetic crystallizer (EMC) mounted at Magnetic Hydrodynamics Scientific and Production Center LLC. In this method of casting, a dispersed structure is obtained with a slight intra-dendritic segregation, which guarantees a high level of mechanical properties. A series of experiments was carried out on continuous pressing at the Conform installation of a batch of $A=12 \mathrm{~mm}$ rod and drawing it to $A 0.5 \mathrm{~mm}$ wire without annealing. To predict the properties of the wire that meet the requirements of TU 1-809-1038-2018, an experimental plan has been drawn up and implemented to determine the dependence of mechanical properties on the exposure time $(\tau)$ and the annealing temperature ( $t$ ) of the wire. As a result of processing the experimental data, regression equations were obtained and graphs of the dependence temporary tensile strength ( $\sigma 8)$ and relative elongation $(\delta)$ on the temperature and holding time, which can be used when annealing $A 0.5 \mathrm{~mm}$ wire from 01417 alloy to obtain the required mechanical properties.
\end{abstract}

Keywords: alloy 01417, electromagnetic crystallizer, aircraft side wires, melt granulation.

Citation: Gorokhov Yu.V., Timofeev V.N., Pervukhin M.V., Belokopytov V.I., Motkov M.M., Erdineev N.B., Kosyachenko I.S., Yakunina O.Ya., Strigin A.S. Manufacturing technology of aluminium wire from alloy 01417 with adjusted level of mechanical properties, J. Sib. Fed. Univ. Eng. technol., 2019, 12(7), 842-851. DOI: 10.17516/1999-494X-0184.

(C) Siberian Federal University. All rights reserved

This work is licensed under a Creative Commons Attribution-NonCommercial 4.0 International License (CC BY-NC 4.0).

* Corresponding author E-mail address: mikhail145@mail.ru 


\title{
Технология изготовления проволоки из сплава 01417
}

\section{с заданным уровнем механических свойств}

\author{
Ю.В. Горохов, В.Н. Тимофеев, М.В. Первухин, \\ В.И. Белокопытов, М.М. Мотков, Н.Б. Эрдынеев, \\ И.С. Косяченко, О.Я. Якунина, А.С. Стригин \\ Сибирский федеральный университет \\ Россия, 660041, Красноярск, пр. Свободный, 79
}

В 70-е годы прошлого столетия отечественными исследователями был разработан сплав алюминия с 7 \% редкоземельных элементов (РЗМ), которые при скоростях охлаждения расплава до $10^{4}$ град/с диспергируются в интерметаллидные фазы, значительно повышаюшие жаропрочность, коррозионную стойкость и свариваемость готовых полуфабрикатов для проводникового материала. Для обеспечения скоростей охлаждения расплава до $10^{4}$ град/с в те годы применялось иентрифугирование гранул в воду. Для повышения эффективности этого передела была применена опытно-промышленная линия для непрерывного прессования гранул методом Конформ. Разработан способ получения из сплавов системы Al-PЗМзаготовок малого сечения с измельченной структурой путем непрерывного литья прутковых заготовок малых сечений (Ø8-12 мм) в электромагнитный кристаллизатор (ЭМК), смонтированный в ООО «НПЦ Магнитной гидродинамики», при этом достигается получение дисперсной структуры с незначительной внутридендритной ликваџией, гарантирующей высокий уровень механических свойств. Проведена серия опытов по непрерывному прессованию на установке Конформ партии отлитого в ЭМК прутка $\varnothing 12$ мм и его волочения на проволоку $\varnothing 0.5$ мм без отжигов. Для прогнозирования свойств проволоки, соответствующих требованиям ТУ 1-809-1038-2018, составлен и реализован план эксперимента по определению зависимости механических свойств

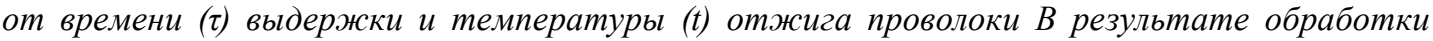
экспериментальных данных получены уравнения регрессии и построены графики зависимости

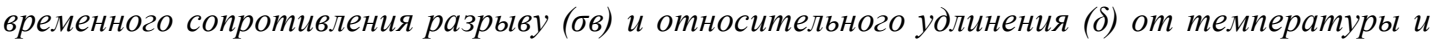
времени выдержки, которые можно использовать при отжиге проволоки $\varnothing 0,5$ мм из сплава 01417 для получения требуемых механических свойств.

Ключевые слова: сплав 01417, электромагнитный кристаллизатор, бортовые провода самолетов, гранулирование расплава.

\section{Введение}

В 70-е годы прошлого столетия отечественными исследователями был разработан сплав алюминия с 7 \% редкоземельных элементов (РЗМ), которые при скоростях охлаждения расплава до $10^{4}$ град/с диспергируются в интерметаллидные фазы, значительно повышающие жаропрочность, коррозионную стойкость и свариваемость готовых полуфабрикатов для проводникового материала. Благодаря этому сплав применяется для изготовления бортовых проводов самолетов, работающих при температурах от минус 50 до $250{ }^{\circ} \mathrm{C}$ взамен проводов с медной жилой [1].

\section{Технология получения проволоки из гранулированного расплава}

Для обеспечения скоростей охлаждения расплава до $10^{4}$ град/с вначале применялось центрифугирование гранул в воду [1]. Технологическая схема производства проволоки из гранульного материала представлена на рис. 1. Узким местом этой схемы является получение из гранул

$$
-843-
$$


прессованного прутка для дальнейшего волочения проволоки. Этот передел состоит из получения брикета штамповкой гильзы, заполненной гранулами, его обточки и прессования, что требовало значительных трудозатрат. При этом отходы металла в стружку и в пресс-остаток составляли $30-40 \%$.

Для повышения эффективности производства проволоки была спроектирована опытнопромышленная линия для непрерывного прессования методом Конформ прутка диаметром 9 мм, смонтированная на участке гранулирования цеха № 8 КраМЗа (рис. 2). Привод колеса диаметром 400 мм с трапецеидальной кольцевой канавкой поперечным сечением 350 мм [2] осуществлялся двигателем постоянного тока мощностью 75 кВт через цилиндрический редуктор с крутящим моментом на выходном валу 60 кНм. Для подачи гранул в ручей колеса использовался бункер. Скорость вращения колеса составляла 10-12 об/мин, при этом температура металла в очаге деформации достигала $420-450^{\circ} \mathrm{C}$, что позволяло вести процесс непрерывного

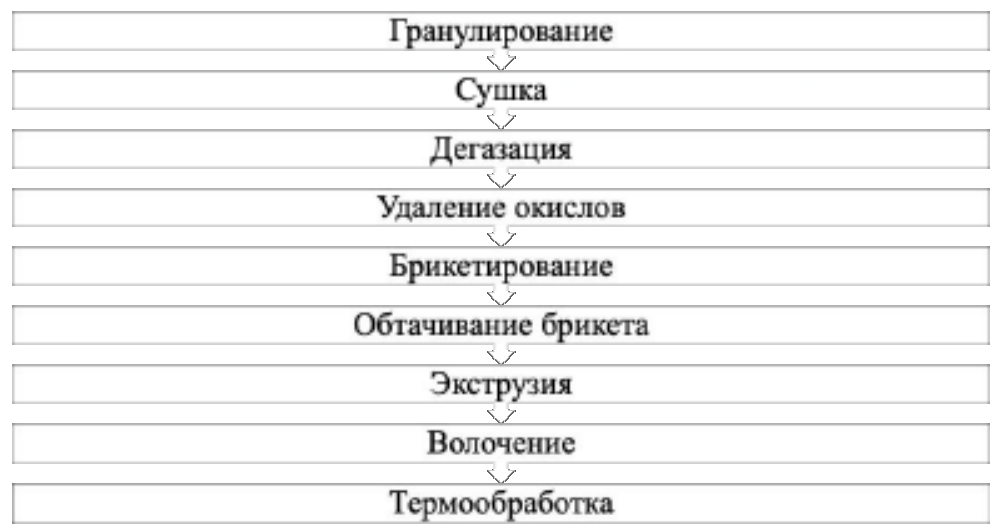

Рис. 1. Технологическая схема получения проволоки из гранул

Fig. 1. Technological scheme for producing wire from granules

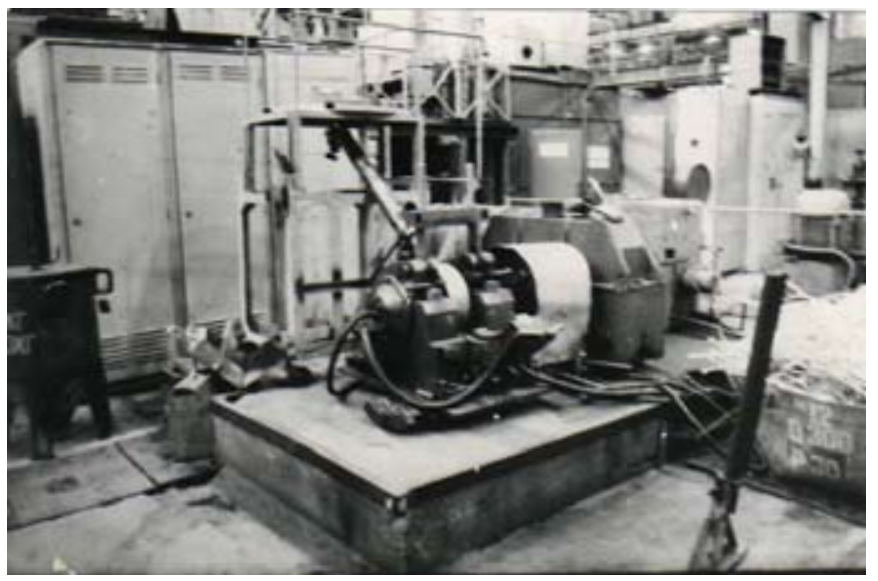

Рис. 2. Установка Конформ на участке по изготовлению прутков из гранулированных алюминиевых сплавов

Fig. 2. Conform installation at the site for the production of bars from granular aluminum alloys 
прессования без предварительного нагрева гранул. Установка снабжена системой принудительного охлаждения рабочего колеса и башмака проточной водой. Отпрессованный пруток поступал на моталку для смотки в бухту [3].

Внедрение в производство установки непрерывного прессования гранул позволило значительно повысить технико-экономические показатели процесса изготовления прутка: производительность, выход годного и т.д., и уменьшить его себестоимость в 5-6 раз.

Однако волочение из прессованного прутка проволоки малого диаметра, необходимой для производства бортовых проводов, проходило с периодическими обрывами из-за наличия внутренних дефектов заготовки, связанных с наследственным влиянием поверхностей гранул [4].

\section{Технология получения проволоки}

\section{из заготовок малого сечения с измельченной структурой}

Для получения из сплавов системы Al-PЗМ заготовок малого сечения с измельченной структурой, которые были бы свободны от недостатков материалов, получаемых методами гранульной технологии, предложено использование непрерывного литья прутковых заготовок малых сечений в электромагнитный кристаллизатор (ЭМК) [5], смонтированный в ООО «НПЦ Магнитной гидродинамики». Литьем длинномерных прутков малого сечения ( $\varnothing 8-12$ мм) из сплава 01417 в ЭМК достигается получение дисперсной структуры с незначительной внутридендритной ликвацией, гарантирующей высокий уровень механических свойств [6].

При волочении из них проволоки на предприятиях ООО «Авиаль», АО ««Электрокабель» Кольчугинский завод» и ООО «Альянс 2008» было установлено, что без предварительного гомогенизирующего отжига волочение слитков сплава 01417 даже с небольшой степенью дендритной ликвации затруднено.

В лабораторных условиях был подобран режим гомогенизационного отжига слитков (550 ${ }^{\circ} \mathrm{C}$, время выдержки 4-5 ч), после которого на месте бывших эвтектических колоний в результате вырождения эвтектики образовались скопления дисперсных алюминидов РЗМ. В результате была устранена внутрикристаллитная ликвация и понижен уровень внутренних напряжений в металле, что обеспечило условия для получения проволоки $\varnothing 0,5$ мм по технологической схеме, представленной в табл. 1.

В заготовке, полученной литьем в ЭМК, наблюдается неравномерность размеров поперечного сечения, что является причиной обрывов на первом переходе, а при волочении на $\varnothing 0,5$ мм применяется три промежуточных отжига в сочетании с гомогенизацией заготовки.

На поставку проволоки для проводов выпущено техническое условие ТУ 1-809-1038-96. В соответствии с ним временное сопротивление разрыву в отожженном состоянии должно составлять не менее 157 МПа, в нагартованном состоянии - не менее 196 МПа, относительное удлинение не менее 8 и не менее $5 \%$ соответственно. Результаты испытания механических свойств готовой проволоки показали, что их уровень не всегда соответствует этим требованиям.

\section{Технология получения проволоки}

\section{с применением непрерывного прессования прутковой заготовки}

Из анализа вышеприведенных технологических схем следует, что для устранения присущих им недостатков целесообразно при производстве проволоки из алюминиевого сплава

$$
-845-
$$


Таблица 1. Технологическая схема изготовления проволоки $\varnothing 0,5$ мм из заготовки, полученной литьем в электромагнитный кристаллизатор

Table 1. Technological scheme for the manufacture of wire $\varnothing 0,5 \mathrm{~mm}$ from a workpiece obtained by casting in an electromagnetic mold

\begin{tabular}{|c|l|c|}
\hline \multicolumn{2}{|c|}{ Сплав 01417} & $\begin{array}{c}\text { Степень } \\
\text { деформации, \% }\end{array}$ \\
\hline $\begin{array}{c}\text { Гомогенизация } \\
\text { заготовки }\end{array}$ & $\begin{array}{l}\mathrm{T}_{\text {гом }} 550{ }^{\circ} \mathrm{C}, \text { охлаждение медленное с печью, далее на воздухе. } \\
\text { Выход на режим 3-4 ч, по закладной термопаре, выдержка 5 ч }\end{array}$ & 82,6 \\
\hline Волочение & $12 \rightarrow 10,9 \rightarrow 10,0 \rightarrow 9,0 \rightarrow 8,0 \rightarrow 7,0 \rightarrow 6,0 \rightarrow 5,0$ & \\
\hline Отжиг & $\begin{array}{l}\mathrm{T}_{\text {отж }} 420^{\circ} \mathrm{C}, \text { охлаждение на воздухе. Выход на режим 4 ч, выдержка } \\
1 \text { ч }\end{array}$ & 84,0 \\
\hline Волочение & $5,0 \rightarrow 4,45 \rightarrow 4,0 \rightarrow 3,6 \rightarrow 3,0 \rightarrow 2,6 \rightarrow 2,25 \rightarrow 2,0$ & 75,0 \\
\hline Отжиг & $\begin{array}{l}\mathrm{T}_{\text {отж }} 420^{\circ} \mathrm{C}, \text { охлаждение на воздухе. Выход на режим 4 ч, выдержка } \\
1 \text { ч }\end{array}$ & \\
\hline Волочение & $2,0 \rightarrow 1,8 \rightarrow 1,6 \rightarrow 1,4 \rightarrow 1,2 \rightarrow 1,0$ & 75,0 \\
\hline Отжиг & \begin{tabular}{l}
$\mathrm{T}_{\text {отж }} 420^{\circ} \mathrm{C}$, охлаждение на воздухе. Выход на режим 4 ч, выдержка \\
\hline ч
\end{tabular} & \\
\hline Волочение & $1,0 \rightarrow 0,9 \rightarrow 0,8 \rightarrow 0,73 \rightarrow 0,66 \rightarrow 0,59 \rightarrow 0,54 \rightarrow 0,5$ & \\
\hline Отжиг & & \\
\hline
\end{tabular}

01417 применить схему, включающую литье в ЭМК заготовки и ее непрерывное прессование на установке Конформ.

Для проведения серии экспериментов по исследованию режимов непрерывного прессования отлитого в ЭМК прутка $\varnothing 12$ мм для волочения проволоки $\varnothing 5$ мм использовалась установка [7], смонтированная в лаборатории ООО «НПЦ Магнитной гидродинамики». Прессовый узел и технические характеристики установки приведены на рис. 3 и в табл. 2.

Методика проведения экспериментов и полученные результаты по исследованию режимов непрерывного прессования прутка $\varnothing 12$ мм подробно описаны в работах [8, 9].

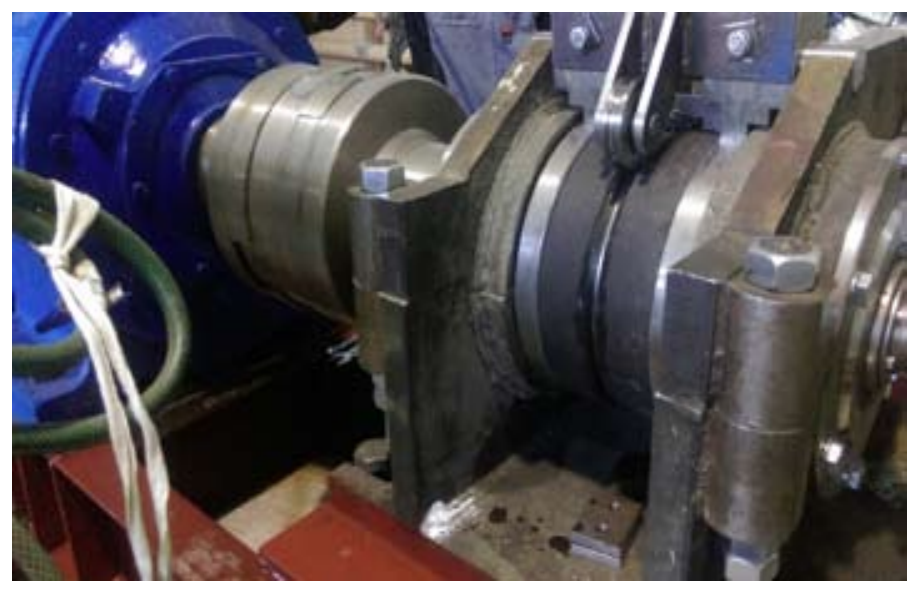

Рис. 3. Опытная установка Конформ ООО «НПЦ Магнитной гидродинамики» [7]

Fig. 3. Pilot installation of Conform LLC "SPC of Magnetic Hydrodynamics” [7] 
Таблица 2. Технические характеристики опытной установки Конформ

Table 2. Specifications pilot plant Conform

\begin{tabular}{|l|c|c|}
\hline \multicolumn{1}{|c|}{ Параметры } & Единицы измерения & Значения \\
\hline Радиус колеса по дну калибра & мм & 22 \\
\hline Мощность электродвигателя & кВт & 24910 \\
\hline Момент на выходном валу & $\mathrm{H} \cdot \mathrm{м}$ & 8 \\
\hline Частота вращения выходного вала & об/мин & \\
\hline
\end{tabular}

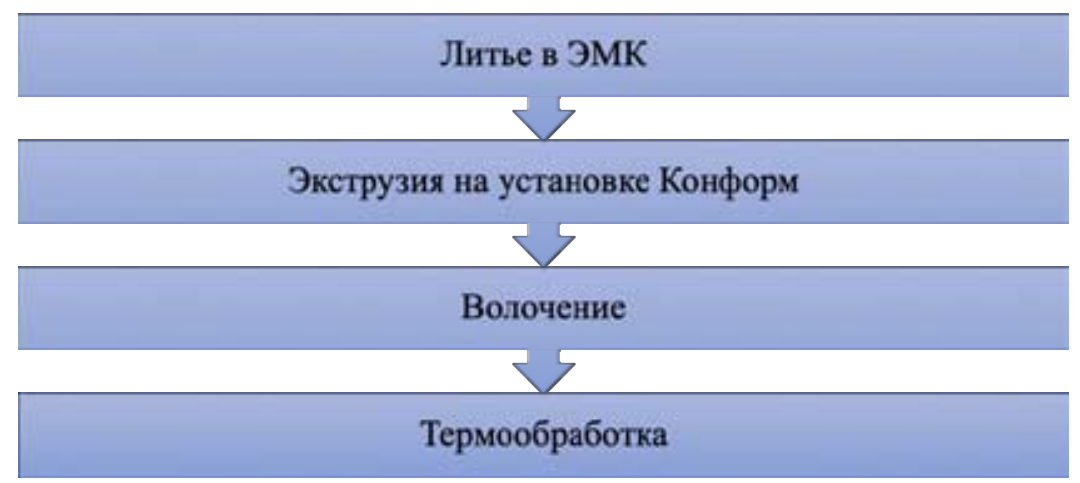

Рис. 4. Технологическая схема получения проволоки из сплава 01417

Fig. 4. Technological scheme for producing wire from alloy 01417

В дальнейшем отпрессованную заготовку без гомогенизации подвергали волочению на $\varnothing 0,5$ мм в условиях ООО «Альянс 2008» за 19 проходов без промежуточных отжигов проволоки. Технологическая схема изготовления проволоки, включающая литье прутка в ЭМК, непрерывное прессование на опытной установке Конформ и волочение, показана на рис. 4.

Следует заметить, что в этой схеме отсутствует отжиг отпрессованного прутка перед волочением.

\section{Эксперименты по исследованию режимов отжига проволоки}

Для обеспечения свойств проволоки $\varnothing 0,5$ мм, полученной по указанной схеме, соответствующих требованиям ТУ-1-809-1038-2018, можно использовать два варианта. Первый - перед заключительными проходами волочения проволоки провести промежуточный отжиг с последующим упрочнением таким образом, чтобы временное сопротивление разрыву и относительное удлинение находились в пределах нормы для нагартованного состояния. Второй - выявить режимы финишного отжига на $\varnothing 0,5$ мм, при которых временное сопротивление разрыву и относительное удлинение находились бы в пределах нормы для нагартованного и отожженного состояний.

Очевидно, что второй вариант предпочтительнее первого. Поэтому проведена серия опытов для построения регрессионной модели зависимости временного сопротивления разрыву $\left(\sigma_{\text {в }}\right)$ и относительного удлинения $(\delta)$ от времени $(\tau)$ выдержки и температуры $(\mathrm{t})$ отжига прово-

$$
-847-
$$


Таблица 3. Условия эксперимента

Table 3. Experimental conditions

\begin{tabular}{|l|c|c|}
\hline \multicolumn{1}{|c|}{ Уровень фактора } & $\mathrm{t},{ }^{\circ} \mathrm{C}$ & $\tau$, мин. \\
\hline Основной $\mathrm{Xi}=0$ & 500 & 60 \\
\hline Интервал варьирования $\Delta \mathrm{xi}$ & 50 & 20 \\
\hline Нижний $\mathrm{Xi}=-1$ & 450 & 40 \\
\hline Верхний Хi $=+1$ & 550 & 80 \\
\hline Кодовые обозначения & $\mathrm{X} 1$ & $\mathrm{X} 2$ \\
\hline
\end{tabular}

Таблица 4. План эксперимента $\sigma_{\mathrm{B}}=\sigma_{\mathrm{B}}(t, \tau)$

Table 4. Experiment Plan $\sigma_{\mathrm{B}}=\sigma_{\mathrm{B}}(t, \tau)$

\begin{tabular}{|c|c|c|c|c|c|c|c|c|c|c|}
\hline \multirow{2}{*}{$\begin{array}{l}\text { Номер } \\
\text { опыта }\end{array}$} & \multirow[t]{2}{*}{$X_{1}$} & \multirow[t]{2}{*}{$X_{2}$} & \multirow[t]{2}{*}{$X_{1} X_{2}$} & \multicolumn{3}{|c|}{$\begin{array}{c}\text { Параллельные опыты } \\
\sigma_{\text {в }}, \text { МПа } \\
\end{array}$} & \multirow[t]{2}{*}{$\bar{y}_{j}$} & \multirow[t]{2}{*}{$\sigma_{j}^{2}$} & \multirow[t]{2}{*}{$\hat{y}_{j}$} & \multirow[t]{2}{*}{$\left(\hat{y}_{j}-\bar{y}_{j}\right)^{2}$} \\
\hline & & & & $y_{1}$ & $y_{2}$ & $y_{3}$ & & & & \\
\hline 1 & + & + & + & 210 & 210 & 215 & 211.7 & 8.3 & 211.7 & 0.00 \\
\hline 2 & - & - & + & 221 & 212 & 208 & 213.7 & 44.3 & 213.7 & 0.00 \\
\hline 3 & + & - & - & 171 & 175 & 171 & 172.3 & 5.3 & 172.3 & 0.00 \\
\hline 4 & - & + & - & 203 & 204 & 203 & 203.3 & 0.3 & 203.3 & 0.00 \\
\hline$\sum$ & & & & & & & & 58.3 & & \\
\hline 5 & 0 & 0 & 0 & 199 & 202 & 201 & 200.7 & 2.3 & 200.3 & 0.17 \\
\hline
\end{tabular}

локи после волочения на $\varnothing 0,5$ мм. Результаты предварительных опытов позволили определить интервалы варьирования этих факторов: $\mathrm{t}=450-550{ }^{\circ} \mathrm{C} ; \tau=40-80$ мин.

В табл. 3 приведены интервалы варьирования для проведения опытов для составления матрицы полного факторного эксперимента.

Временное сопротивление разрыву отожженных образцов определяли в соответствии с ГОСТ 10446 на машине LFM-100 в соответствии с ГОСТ 10446. Полученные результаты занесены в табл. 4.

\section{Результаты обработки экспериментальных данных}

В результате обработки по стандартной методике [10] данных табл. 4 получено уравнение регрессии:

$$
\hat{y}=200,25-8,25 X_{1}+12,42 X_{2}+7,25 X_{3},
$$

которое при переходе от кодированных значений факторов к натуральным принимает вид

$$
\begin{gathered}
\sigma_{B}=200,25-8,25\left(\frac{t-500}{50}\right)+12,42\left(\frac{\tau-60}{20}\right)+7,25\left(\frac{t-500}{50}\right)\left(\frac{\tau-60}{20}\right)= \\
=462,99-0,6 t-3,000415 \cdot \tau+0,00725 \cdot t \cdot \tau . \\
-848-
\end{gathered}
$$


Аналогичным образом получено уравнение регрессии, показывающее зависимость относительного удлинения ( $\delta$ ) от времени $(\tau)$ выдержки и температуры (t) отжига проволоки.

$$
\begin{aligned}
& \delta=9,65+1,25\left(\frac{t-500}{50}\right)-2,4\left(\frac{\tau-60}{20}\right)+2,133\left(\frac{t-500}{50}\right)\left(\frac{\tau-60}{20}\right)= \\
& =67,89-0,1022 t-1,1735 \cdot \tau+0,00211 \cdot t \cdot \tau .
\end{aligned}
$$

По экспериментальным и рассчитанным по уравнениям регрессии данным на рис. 5 построен график зависимости временного сопротивления разрыву (бв) и относительного удлинения ( $\delta$ ) проволоки $\varnothing 0,5$ мм от температуры и времени выдержки отжига.

На графиках рис. 5 видно, что расхождение теоретических и экспериментальных данных составляет не более $5 \%$. На основании этого делаем вывод, что уравнения регрессии можно использовать для прогнозирования свойств проволоки при финишном отжиге. Результаты исследования можно рекомендовать для промышленного использования при изготовлении проволоки из сплава 01417, соответствующей требованиям ТУ 1-809-1038-2018.

\section{Заключение}

1. На основании анализа технологических схем получения проволоки из гранулированных сплавов выявлено, что при этом требуется большое количество технологических переделов, а волочение проволоки малого диаметра не стабильно вследствие периодических обрывов из-за внутренних дефектов, связанных с наследственным влиянием поверхностей гранул.

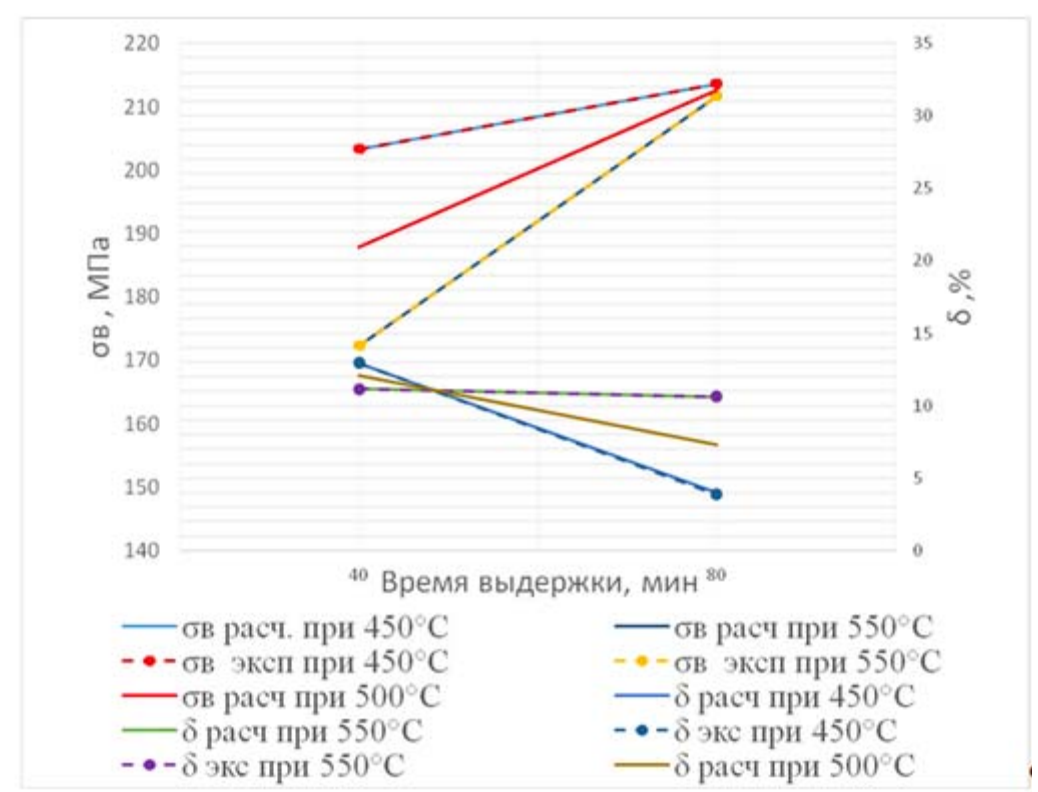

Рис. 5. График зависимости временного сопротивления разрыву (бв) и относительного удлинения $(\delta)$ от температуры и времени выдержки проволоки $\varnothing 0,5$ мм при отжиге

Fig. 5. Graph of the dependence of the temporary tensile strength (бв) and elongation $(\delta)$ on the temperature and the exposure time of the wire $\varnothing 0.5 \mathrm{~mm}$ during annealing 
2. Проанализирован способ получения проволоки из заготовки, полученной методом литья в электромагнитный кристаллизатор. Пруток $\varnothing 12$ мм имеет разнотолщинность по всей длине полуфабриката от 10 до 14 мм, что приводит к обрывам при волочении проволоки на первых проходах. При волочении проволоки $\varnothing 0,5$ мм из прутковой заготовки 12 мм за 19 переходов требуется три промежуточных отжига, что увеличивает себестоимость продукции.

3. В результате использования математического планирования эксперимента получены уравнения регрессии и построены графики зависимости временного сопротивления разрыву $\left(\sigma_{\mathrm{B}}\right)$ и относительного удлинения ( $\left.\delta\right)$ от температуры и времени выдержки, которые можно использовать при отжиге проволоки $\varnothing 0,5$ мм из сплава 01417 для получения уровня механических свойств, требуемых нормами ТУ 1-809-1038-96.

\section{Благодарности}

Исследование выполнено при финансовой поддержке Российского фонда фундаментальных исследований, Правительства Красноярского края, Красноярского краевого фонда науки и ООО «Научно-производственный центр магнитной гидродинамики» в рамках научного проекта № 18-48-242023 «Исследование динамических характеристик турбулентных течений расплава при электромагнитной кристаллизации и влияния ее на структуру и свойства непрерывнолитых заготовок малого сечения из новых алюминиевых сплавов для производства тонкой проволоки авиационно-космического назначения».

\section{Список литературы}

[1] Горбунов Ю.А. Роль и перспективы редкоземельных металлов в развитии физикомеханических характеристик и областей применения деформируемых алюминиевых сплавов. Журнал Сибирского федерального университета. Техника и технологии, 2015, 8(7), 636645 [Gorbunov Yu.A. Role and prospects of rare-earth metals in the development of physical and mechanical characteristics and applications of deformable aluminum alloys. J. Sib. Fed. Univ. Eng. technol., 2015, 8(7), 636-645 (in Russian)]

[2]. Северденко В.П., Шепельский Н.В., Горбунов Ю.А., Жилкин В.3. Прокатка гранул алюминиевых сплавов. Наука и техника, Минск, 1978, 216 с. [Severdenko V.P., Shepelsky N.V., Gorbunov Yu.A., Zhilkin V.Z. Pellet rolling of aluminum alloys. Science and Technology. Minsk, 1978, 216 p. (Russian)]

[3] Шеркунов В.Г., Горохов Ю.В., Константинов И.Л. и др. Использование способа Конформ для переработки стружки из алюминиевых сплавов. Известия высших учебных заведений. Цветная металлургия, 2015, 3, 60-63 [Sherkunov V.G., Gorokhov Yu.V., Konstantinov I.L. and others. Using the Conform method for processing chips from aluminum alloys. News of higher educational institutions. Non-ferrous metallurgy, 2015, 3, 60-63 (in Russian)]

[4] Горохов Ю.В., Корнилов В.Н., Константинов И.Л. и др. Линия для непрерывного прессования электротехнической проволоки из вторичного алюминия. Новые материалы и технологии. Ч.1. Металлические материалы и методы их обработки. М.: МГАУ, 1994. С. 118. [Gorokhov Yu.V., Kornilov V.N., Konstantinov I.L. et al. Line for continuous extrusion of electrical wire from secondary aluminum. New materials and technologies. Part 1. Metallic materials and methods for their processing. M., MGAU, 1994. P. 118 (in Russian)]

$$
-850-
$$


[5] Гецелев 3.Н. и др. Непрерывное литье в электромагнитный кристаллизатор. М.: Металлургия, 1983. 152 c. [Getselev Z.N. Continuous casting in an electromagnetic mold. M.: Metallurgy, 1983. 152 p. (in Russian)]

[6] Стеценко В.Ю. Механизмы процесса кристаллизации металлов и сплавов. Литье и металлургия. БНТУ. Минск, 2013, 1,48-54 [Stetsenko V.Yu. Mechanisms of the process of crystallization of metals and alloys. Casting and Metallurgy. BNTU, Minsk, 2013, 1, $48-54$ (in Russian)]

[7] Горохов Ю.В., Тимофеев В.Н., Беляев С.В., Авдулов А.А., Усков И.В., Губанов И.Ю., Авдулова Ю.С., Иванов А.Г. Прессовый узел установки Conform для непрерывного прессования цветных металлов Изв. вузов. Цвет. металлургия, 2017, 4, 69-75 [Gorokhov Yu.V., Timofeev V.N., Belyaev S.V., Avdulov A.A., Uskov I.V., Gubanov I.Yu., Avdulova Yu.S., Ivanov A.G. The press unit of the Conform installation for continuous pressing of non-ferrous metals, Izv. universities. Color. Metallurgy, 2017, 4, 69-75 (in Russian)]

[8] Авдулов А.А., Сергеев Н.В., Гудков И.С., Тимофеев В.Н., Горохов Ю.В., Авдулова Ю.С. Разработка технологии производства проволоки из специальных алюминиевых сплавов на основе способа литья в электромагнитный кристаллизатор и непрерывного прессования способом Конформ. Журнал Сибирского федерального университета. Техника и технологии, 2017, 10(1), 85-94 [Avdulov A.A., Sergeev N.V., Gudkov I.S., Timofeev V.N., Gorokhov Yu.V., Avdulova Yu.S. Development of technology for the production of wire from special aluminum alloys based on the method of casting in an electromagnetic mold and continuous pressing by the Conform method, J. Sib. Fed. Univ. Eng. technol., 2017, 10(1), 85-94 (in Russian)]

[9] Горохов Ю.В., Тимофеев В.Н., Беляев С.В., Усков И.В., Губанов И.Ю., Гудков И.С. Прессование слитков из сплава 01417, полученных в электромагнитном кристаллизаторе на установке Conform. Изв. вузов. Цвет. металлургия, 2018, 4, 53-59 [Gorokhov Yu.V., Timofeev V.N., Belyaev S.V., Uskov I.V., Gubanov I.Yu., Gudkov I.S. The pressing of ingots from alloy 01417 obtained in an electromagnetic crystallizer at a conform installation. Izv. universities. Color. Metallurgy, 2018, 4, 53-59 (in Russian)]

[10] Белокопытов В.И. Статистические методы управления качеством металлопродукции. Красноярск: Сиб. федер. ун-т, 2011. 108 с. [Belokopytov V.I. Statistical methods of quality management of metal products. Krasnoyarsk, Sib. feder. un-t, 2011. 108 p. (in Russian)] 